\title{
DEVELOPMENT OF A VISCOELASTIC CONTINUUM DAMAGE MODEL FOR CYCLIC LOADING
}

\author{
R.W. Sullivan
}

Department of Aerospace Engineering, Mississippi State University

\begin{abstract}
A previously developed spectrum model for linear viscoelastic behavior of solids is used to describe the rate-dependent damage growth of a polymer matrix composite under cyclic loading. Through the use of the iterative solution of a special Volterra integral equation, the cyclic strain history is described. Damage evolution in the body is described through the use of a rate-type evolution law which uses a pseudo strain to express the viscoelastic constitutive equation with damage. The resulting damage function is used to develop a residual strength model. The peak values of the analytic cyclic strain history as well as the residual strength model predictions agree well with the experimental data.
\end{abstract}

\section{INTRODUCTION}

Fiber reinforced polymer composites exhibit time dependent behavior, due primarily to the matrix component. The viscoelastic response can become accelerated due to a combination of both creep and cyclic loading [1]. Kumar and Talreja [2] describe the various damage mechanisms associated with both elastic and viscoelastic laminates as the applied stress or number of fatigue cycles increases. Various damage models are used to quantify the ensuing damage and thereby predict properties of interest. In this paper, the viscoelastic nature of the composite is represented through the use of a pseudo strain and the damage state is described by a continuous damage variable that uses the strain response of the composite laminate.

\section{VISCOELASTIC FATIGUE MODEL}

Previously, a distribution function was used to develop the time dependent response and properties of interest of an isotropic solid under a constant loading condition [3]. The resulting strain response has been extended in this study to model the complete strain history of a solid under cyclic stress. The resulting strain as a function of time is given by

$$
\varepsilon(\mathrm{t})-\frac{\mathrm{n}}{\pi} \int_{0}^{\mathrm{t}} \mathrm{e}_{11}(\mathrm{t}-\tau)\left\{\int_{\mathrm{n}_{\mathrm{o}}}^{\infty} \frac{\cos \left(\mathrm{z}-\mathrm{n}_{\mathrm{o}} \mathrm{t}\right)}{\mathrm{z}} \mathrm{dz}\right\} \mathrm{d} \tau=\frac{\sigma(\mathrm{t})}{\mathrm{E}_{\mathrm{e}}}
$$

where

$$
\sigma(t)= \begin{cases}\sigma_{0}, & \text { uniaxial creep } \\ \sigma_{\mathrm{m}}(1+\mathrm{A} \sin (\omega \mathrm{t})) & \text { cyclic loading }\end{cases}
$$

and $\omega$ is the angular frequency of the cyclic test. Equation (1) is a special Volterra integral equation and it is solved by the method of iteration. The viscoelastic parameters $n$ and $n_{o}$ are determined from the experimental creep strain, enabling the determination of other properties of interest, such as the time dependent modulus which is given as

$$
E(t)=E_{e}\left[\left(1-\frac{n}{2 n_{0}}\right)+\frac{n}{\pi n_{o}} \int_{n_{0} t}^{\infty} \frac{\sin \left(z-n_{0} t\right)}{z} d z\right] .
$$

\section{THE CONSTITUTIVE MODEL USING PSEUDO STRAIN}

In general viscoelastic solids satisfy an integral form of the constitutive equation such as the BoltzmannVolterra equation and much of the theory of linear viscoelasticity uses the Principle of Correspondence to transition from the analytical elastic models to the time dependent models as has been done to develop the model in Eq. (1). An alternate method [4] that is used involves the use of pseudo variables so that the viscoelastic stress-strain relationship can be described using elastic-like equations. The concept of pseudo strain is developed through the equation

\footnotetext{
*Department of Aerospace Engineering, Mississippi State University, Miss. State, MS 39762

Sullivan@ae.msstate.edu.
} 


$$
\varepsilon^{\mathrm{R}}=\frac{1}{\mathrm{E}_{\mathrm{R}}} \int_{0}^{\mathrm{t}} \mathrm{E}(\mathrm{t}-\tau) \frac{\mathrm{d} \varepsilon}{\mathrm{d} \tau} \mathrm{d} \tau
$$

where $\varepsilon^{R}$ is the pseudo strain and $\varepsilon$ is the actual strain, while $E(t)$ is the time-dependent modulus and $E_{R}$ is a reference modulus. Equation (4) can also be expressed for a viscoelastic material in a form similar to the Hooke's Law of linear elasticity as

$$
\sigma(t)=M(t) \varepsilon^{R}(t)
$$

where $\mathrm{M}(\mathrm{t})$ is a general constitutive function. The simplicity of this description is that the integral form of the constitutive equation has now been described by the pseudo strain which contains the memory aspect of the material. Using Eq. (3) in Eq. (4), the pseudo strain for any strain rate (which is calculated from the cyclic strain response) can be written as

$$
\varepsilon^{R}=\frac{E_{e}}{E_{R}} \int_{0}^{t}\left[\left(1-\frac{m}{2}\right)+\frac{m}{\pi} \int_{n_{0} t}^{\infty} \frac{\sin \left(z-n_{0} t\right)}{z} d z\right] \frac{d \varepsilon}{d \tau} d \tau
$$

\section{DEVELOPMENT OF DAMAGE MODEL}

A continuum damage mechanics approach is used to formulate the damage function. Based on the concept of the load bearing area decreasing with increasing damage, the damage factor is defined in terms of a "net stress" as [5]

$$
\sigma^{\prime}=\frac{\sigma}{1-\mathrm{S}}
$$

where $\sigma$ is the applied stress. Kachanov's damage growth equation [6] is solved and the solution is

$$
S(t)=1-\left\{1-C(\eta+1) \int_{0}^{t}[\sigma(t)]^{\eta} d t\right\}^{\frac{1}{\eta+1}} .
$$

To determine the material constants, $\mathrm{C}$ and $\eta$, the case of constant loading is evaluated at rupture, i.e., $\mathrm{S}=$ 1 , resulting in an expression for the creep rupture time. Beginning with the simplest form of the free energy of deformation (Helmholtz), the strain energy density function $\mathrm{W}$ is first defined to be a function of the pseudo strain and implicitly, of a damage variable $\mathrm{S}$ as

$$
\mathrm{W}=\frac{1}{2} \mathrm{M}(\mathrm{S})\left(\varepsilon^{\mathrm{R}}\right)^{2}
$$

where $\mathrm{M}(\mathrm{S})$ is a constitutive function of damage. The components of the stress may be obtained by taking the first derivative of $\mathrm{W}$ with respect to the pseudo strain, thereby obtaining Eq. (5). Since $\mathrm{S}$ is a function of time, the constitutive function $M(S)$ is also a function of time, i.e., $M(S)=M[S(t)]=M(t)$. Having defined the stress function by Eq. (5) and solving Eq. (8) for the term $C(\eta+1)$ for the rupture condition, the damage function can now be expressed in terms of the pseudo strain as

$$
S(t)=1-\left\{1-\frac{\int_{0}^{t}\left[M(t) \varepsilon^{R}(t)\right]^{\eta} d t}{\int_{0}^{t_{R}}\left[M(t) \varepsilon^{R}(t)\right]^{\eta} d t}\right\} .
$$

For the present study, $\mathrm{M}(\mathrm{t})$ is taken to be equal to $\mathrm{E}(\mathrm{t})$ and determined from Eq. (3). It is observed that the damage function inherently contains information about the degradation of strength in the material and its 
composition is seen to contain a "pseudo stress", i.e., $M(t) \varepsilon^{\mathrm{R}}(\mathrm{t})$. Taking guidance from the results of the damage function in Eq. (10), a form for the remaining or residual strength is proposed by forming a factor $\operatorname{Fr}(\mathrm{t})$ as given by

$$
\operatorname{Fr}(t)=\left\{1-\frac{\beta \int_{0}^{t}\left|\varepsilon^{R}(t)\right|^{\eta} d t}{\int_{0}^{t f}\left|\varepsilon^{R}(t)\right|^{\eta} d t}\right\}^{\frac{1}{\eta+1}}
$$

for a time in the range $\left[0, t_{\mathrm{f}}\right]$ where $\mathrm{t}_{\mathrm{f}}$ is some final time and the parameter $\beta$ is to be determined by comparison with either the experimental or the empirical data. Related to the initial strength of the material $\sigma_{\mathrm{o}}$ and the factor $\operatorname{Fr}(\mathrm{t})$ given in Eq. (11), the residual strength $\sigma_{\mathrm{R}}(\mathrm{t})$ is formulated as

$$
\sigma_{\mathrm{R}}(\mathrm{t})=\sigma_{\mathrm{o}} \operatorname{Fr}(\mathrm{t})
$$

\section{RESULTS}

Data from constant amplitude fatigue loading was available for a polymer composite laminate, for which both fatigue to failure and residual strength tests had been performed. For initial model validation, the composite laminate was regarded as an effective homogenous continuum. Additionally, due to lack of creep data on the same material system, strain at the mean stress level was used for the creep strain history and $\eta$ was set equal to $m$, the viscoelastic parameter. Figure 1 depicts the cyclic strain history compared with the measured peak and mean strains for a final time of 2000 seconds and the inset figure shows a shorter time scale of $t=200$ seconds. The peak values of the response agree well with the measured peak strains. Fig. 2 shows the residual strength behavior, normalized with respect to the initial strength, with the experimental data.

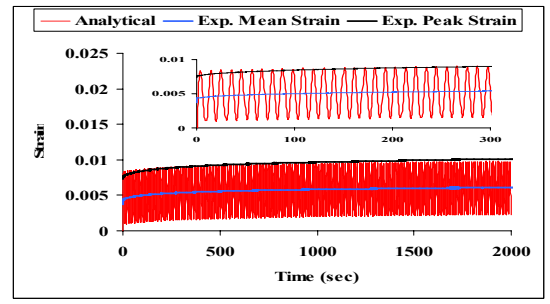

Figure 1. Cyclic Strain Response

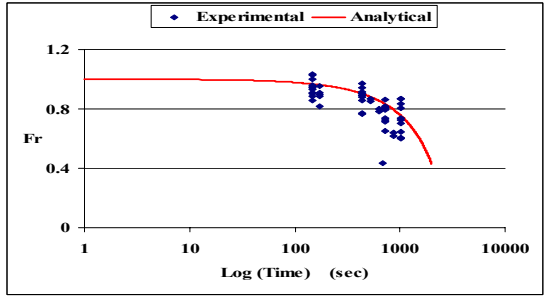

Figure 2. Normalized Residual Strength

\section{CONCLUSIONS}

From an earlier development, a model to describe creep response is modified to simulate the strain response of a viscoelastic material under cyclic loading. The resulting response is used to calculate the pseudo strain that is used to develop a damage model. Based on the results of the damage model, a residual strength function is formulated and compared to the experimental data. There is good agreement between the model predictions and the experimental data.

\section{ACKNOWLEDGEMENTS}

This work was sponsored by the Office of Naval Research (Contract \#: N00014-06-1-0812) through Virginia Tech (VT). Thanks are due to the project technical advisor Mr. B. Jones (NSWC) and the program manager Dr. I. Perez (ONR). The author is also appreciative of the support and test data from Dr. J. Lesko, Dr. S. Case and Mr. N. Post of VT.

\section{REFERENCES}

1. Vinogradov, A.M., Jenkins, C.H.M., Winter, R.M., Long Term Durability of Structural Materials:Proc. Of the Durability Workshop, Berkeley, California, Oct. 26-27, 2000.

2. Kumar, RS., Talreja, R., Mechanics of Materials, 35, 463-480, 2001.

3. Sullivan, R.W., Mechanics of Time Dependent Materials, 10, 215-228, 2006.

4. $\quad$ Park, S., Kim, Y., Schapery, R., Mechanics of Materials, 24, 241-255, 1996.

5. Stigh, U., ASME Journal of Applied Mechanics, 73, 702-704, 2006.

6. Kachanov, L. M., Izv. Akad. Nauk SSR, Otd. Tekhn. Nauk, 8, 26-31, 1958. 